\title{
SPHERICAL CURVES AND THEIR ANALOGUES IN AFFINE DIFFERENTIAL GEOMETRY ${ }^{1}$
}

\author{
ERWIN KREYSZIG AND ALOIS PENDL
}

\begin{abstract}
Necessary and sufficient conditions for curves. in Euclidean space to be spherical are derived in a fashion which can be generalized to affine differential geometry and analogues of those curves. This also includes a discussion of some geometrical aspects in recent papers by $\mathrm{S}$. Breuer, D. Gottlieb, and Y.-C. Wong.
\end{abstract}

1. Introduction. The problem of characterizing classes of ordinary differential equations which can be transformed into equations with constant coefficients was recently considered by S. Breuer and D. Gottlieb [1]. In [2] the authors gave an application to spherical curves by deriving a simple differential equation for the radius of curvature. This result was later used by Y.-C. Wong [9] in connection with his criterion in [8] for a curve to be spherical.

We want to show that the formal approach in [2] has an underlying geometrical idea, which yields the reason for the simplicity of the result in [2], and we shall also give a geometrical motivation and simplified derivation of Wong's result.

Furthermore, that idea can be modified so that it becomes applicable in affine differential geometry. This field was developed by E. Salkowski [6] and others. In a more general and abstract form it has recently become important mainly for functional analytic reasons. In fact, such a "differential geometry of vector spaces" was initiated by E. R. Lorch [4] and R. Nevanlinna [5] and has applications in physics, for instance in the theory of elasticity of anisotropic media. The notion of a sphere does not make sense in that geometry, but we shall define spherical curves in affine space in terms of a property which is characteristic of those curves in Euclidean space. We shall also obtain a condition necessary and sufficient for a curve in affine space to be spherical.

Received by the editors September 24, 1973.

AMS (MOS) subject classifications (1970). Primary 53A05, 53A15.

1 Research supported by a grant from the National Re search Council of Canada.

Copyright $\odot$ 1975. American Mathematical Society 
2. Notations. Modified Frenet formulas. Let $x: J \rightarrow E_{3}$ represent a curve $C$ in Euclidean space $E_{3}$, where $J \subset R$ is any fixed open interval. We always assume that $C$ has a unique tangent and positive curvature $\kappa$ on $J$ and all appearing derivatives exist and are continuous functions of the arc length $s$ of $C$ on $J$. We also assume that the torsion $\tau$ of $C$ is not zero (but shall drop this assumption later). Let $t, p, b$ be the trihedron of $C$, and define $\alpha, \beta, \gamma$ by

$$
d \alpha=\kappa d s, \quad d \beta=\sqrt{\kappa^{2}+\tau^{2}} d s, \quad d \gamma=\tau d s ;
$$

these quantities are usually called the angles of contingence of the tangent, principal normal, and binormal, respectively, and $y$ will play a crucial role in our approach. Setting $\lambda=\kappa / \tau$, we can easily obtain Frenet formulas for derivatives with respect to $\gamma$ (denoted by dots):

$$
\dot{t}=\lambda p, \quad \dot{p}=b-\lambda t, \quad \dot{b}=-p .
$$

3. Differential equation for $\rho$. A curve $C$ is spherical iff there is a point in common with all normal planes of $C$. This holds iff there is a cone $S$ passing through $C$ and such that any generator of $S$ through a point $P \epsilon$ $C$ lies in the normal plane of $C$ at $P$. Clearly, $S$ is a ruled surface which can be represented in the form

$$
y(r, s)=x(s)+r(A(s) p(s)+B(s) b(s)),
$$

where $x$ represents $C$. The surface $S$ is a cone iff there is a function $r$ of $s$ such that

$$
x(s)+v(s) p(s)+w(s) b(s)=k,
$$

where $v(s)=r(s) A(s), w(s)=r(s) B(s)$, and $k$ is a constant vector. This is equivalent to

$$
\dot{x}+\dot{v p}+v \dot{p}+\dot{w} b+w \dot{b}=0 .
$$

Applying (2) and $\dot{x}=t / \tau$, we have

$$
\tau^{-1} t+\dot{v} p+v(b-\lambda t)+\dot{w} b-w p=0 .
$$

Equating the coefficients of $t, p, b$ to zero, we have successively

$$
v=\rho, \quad w=\dot{v}=\dot{\rho}, \quad v+\dot{w}=0 .
$$

The last relation becomes simply

$$
\ddot{\rho}+\rho=0 .
$$

Solutions are of the form 


$$
\rho(s)=R \cos \left(\gamma(s)+\gamma_{0}\right), \quad \gamma(s)=\int_{s_{0}}^{s} \tau(\tilde{s}) d \tilde{s}
$$

where $R$ is the radius of the sphere of $C$. Note that these solutions depend on the torsion. (5) is a necessary and sufficient condition for $C \subset E_{3}$ to be spherical (even when $\tau=0$ for some $s$ ). (4) and (5) were obtained in [2] in an entirely different way, and our derivation shows that the simplicity of the result is achieved because we used the angle of contingence of the binormal.

4. Another natural equation. A more familiar (but more complicated) natural equation of a spherical curve with $\tau \neq 0$ is readily obtained from (4) and (1):

$$
\left(\rho^{\prime} / \tau\right)^{\prime}+\rho \tau=0
$$

where primes denote derivatives with respect to $s$. Of course other ways of deriving (6) can be modified so that they yield (4). For instance, assuming the formula for the centers of curvature, we have

$$
z=x+\rho p+\left(\rho^{\prime} / \tau\right) b=x+\rho p+\dot{\rho} b .
$$

$C$ is spherical iff $z$ is constant; thus

$$
\dot{z}=\dot{x}+\dot{\rho} p+\rho \dot{p}+\ddot{\rho} b+\dot{\rho} \dot{b}=0 \text {. }
$$

From this and (2), equation (4) follows.

5. On theorems by Y.-C. Wong. A curve $C: x(s)$ with nonzero curvature $\kappa$ and the torsion $\tau$ is spherical iff (6) holds. If $\tau=0$ at some $s$, then (6) is no longer applicable. This case was considered by Y.-C. Wong [8], [9] who proved the following two theorems.

I. A curve $C: x \in C^{4}(J), J=\left[s_{1}, s_{2}\right]$, in $E_{3}$ with a unique tangent is spherical iff it satisfies the two conditions:

(i) $\kappa(s)>0$ in $\mathrm{J}$;

(ii) there is a function $f \in C^{\prime}(J)$ such that

$$
f \tau=\rho^{\prime}, \quad f^{\prime}=-\rho \tau \quad(s \in J) .
$$

II. The curve $C$ in Theorem I is spherical iff it satisfies (5).

Note that it is not difficult to see that a spherical curve with a unique tangent has positive curvature and thus a unique trihedron.

Wong proved II by showing that (5) satisfies (i) and (ii) and, conversely, can be obtained from (i) and (ii). In [9] he expresses surprise that this is so. We shall give another proof of I which rests on geometrical arguments and thus 
explains the geometrical background of Wong's approach and the connection between I and II.

A sphere in $E_{3}$ having contact of second order with a curve $C: x(s)$ has center $a=x+\rho p+h b$ where $h$ is arbitrary; cf. [3, p. 54]. If for every $s$ we associate with $C$ such a sphere, these spheres have constant radius $|a-x|$ iff

$$
\rho \rho^{\prime}+b b^{\prime}=0 .
$$

These spheres have contact of third order iff $\rho \kappa^{\prime}+h \kappa \tau=0$; cf. [3, p. 54] which is equivalent to

$$
\rho^{\prime}=b r
$$

From this and (8) we have

$$
\rho \tau+b^{\prime}=0 .
$$

If $r \neq 0$, we get $h=\rho^{\prime} / \tau$, and (9b) yields (6) as a necessary and sufficient condition for $C$ to be spherical. If $r=0$ for some $s$, equations (9a) and (9b) still make sense and are precisely the condition (7) (with $f$ denoted by $h$ ).

Note that we were dealing with osculating spheres, which have contact of third order with the curve, so that Wong's assumption $x \in C^{4}(J)$ is natural, albeit not the weakest one. Note further that $\tau=0$ at an $s_{0}$ implies $\rho^{\prime}=$ 0 at $s_{0}$; cf. (9a); that is, if the osculating plane is stationary $\left(b^{\prime}=0\right)$ and $C$ is spherical, then $\kappa$ must be stationary at that point. This is geometrically understandable.

To explain the connection between I and II in geometrical terms, we may set $h=\dot{H}$ in (9a) and integrate. Then we see that $\rho=H+c$. Hence the function $f$ in (7) is geometrically the derivative of the radius of curvature with respect to $\gamma$. In (9b) we then have

$$
\tau(\rho+\ddot{H})=\tau(\rho+\ddot{\rho})=0,
$$

which gives a reason for the relation between I and II.

6. Some concepts of affine differential geometry. We want to show that the idea of $\S_{3}$ can be generalized to curves in affine space $A_{3}$. For this we shall need a few simple concepts and facts as follows. Affine differential geometry investigates invariants with respect to the group of those affine transformations

$$
x_{j}^{*}=\sum_{k=1}^{3} a_{j k} x_{k}+c_{j} \quad(j=1,2,3)
$$


which are volume-preserving $\left(\operatorname{det}\left(a_{j k}\right)=1\right)$. Such a transformation is said to be equiaffine, and affine differential geometry is also known as equiaffine differential geometry.

Let $x: J \rightarrow A_{3}$ represent a curve $C$ in $A_{3}$, where $J=\left(u_{1}, u_{2}\right) \subset R$ is any fixed open interval. We assume that $x \in C^{4}(J)$ and $|\dot{x} \ddot{x} \ddot{x}| \neq 0$ on $J$, where $\dot{x}=d x / d u$, etc. Then with $C$ we may associate the invariant parameter

$$
\sigma(u)=\int_{u_{1}}^{u}|\dot{x} \ddot{x} \ddot{x}|^{1 / 6} d u^{*} \quad\left({ }^{*}=d / d u^{*}\right)
$$

which is called the affine arc length of $C$ and yields a representation $x(\sigma)$ of $C$. A trihedron of $C$ consists of the tangent vector $t=x^{\prime}$, the affine normal vector $p=x^{\prime \prime}$, and the affine binormal vector $b=x^{\prime \prime \prime}$; here $x^{\prime}=d x / d \sigma$, etc. The vectors $p$ and $b$ span the affine normal plane. The affine Frenet formulas are

$$
t^{\prime}=p, \quad p^{\prime}=b, \quad b^{\prime}=-\tilde{\tau} t-\tilde{\kappa} p .
$$

They involve the invariants

$$
\tilde{\kappa}=\left|x^{\prime} x^{\prime \prime \prime} \cdot x^{\mathrm{iv}}\right|, \quad \tilde{\tau}=-\left|x^{\prime \prime} x^{\prime \prime \prime} x^{\mathrm{iv}}\right|,
$$

which are called the affine curvature and torsion of $C$.

7. Spherical curves in affine space $A_{3}$. The property of spherical curves in $E_{3}$ stated at the beginning of $\$ 3$ suggests the following

Definition. A curve $C \subset A_{3}$ is said to be spherical if all affine normal planes of $C$ pass through a common point in $A_{3}$.

As in $\S_{3}$ this holds iff there is a cone $S \supset C$ such that any generator of $S$ through a point $P \in C$ lies in the affine normal plane of $C$ at $P$. This holds iff there is a function $r$ of $\sigma$ such that in the representation

$$
y(r, \sigma)=x(\sigma)+r(A(\sigma) p(\sigma)+B(\sigma) b(\sigma))
$$

we have

$$
x(\sigma)+v(\sigma) p(\sigma)+w(\sigma) b(\sigma)=k
$$

where $v(\sigma)=r(\sigma) A(\sigma), w(\sigma)=r(\sigma) B(\sigma)$, and $k$ is a constant vector. This is equivalent to

$$
t+v^{\prime} p+\dot{v} p^{\prime}+w^{\prime} b+w b^{\prime}=0 .
$$

Applying (10) and equating to zero the coefficients of the independent vectors $t, p, b$, we have the three conditions 


$$
\widetilde{\tau} w=1, \quad v^{\prime}=\tilde{\kappa} w, \quad v+w^{\prime}=0
$$

A solution is

$$
\tilde{\kappa}=\tilde{\tau}\left(\tilde{\tau}^{\prime} / \tilde{\tau}^{2}\right)^{\prime} .
$$

Performing the indicated differentiation, we could cast this in the form of a Riccati equation, which also appeared in a paper by L. A. Santalo [7] who obtained it in a different way. However, (11) and its derivation, together with suitable differentiability assumptions, give immediately the following remarkable criterion.

Theorem. Let $C: x(u)$ be a curve in affine space $A_{3}$ which is of class $C^{6}(J)$ on some fixed open interval $J$ and satisfies $|\dot{x} \ddot{x} \ddot{x}| \neq 0$ and $\tilde{\tau} \neq 0$ on $J$, where $\tilde{\tau}$ is the affine torsion of $C$. Then $C$ is spherical (definition above) iff

$$
\chi^{\prime \prime}+\tilde{\kappa}(\sigma) \chi=0,
$$

where $\sigma$ and $\tilde{\kappa}$ are the affine arc length and affine curvature of $C$, and $\chi=1 / \tilde{\tau}(\tilde{\tau} \neq 0)$ is the affine radius of torsion of $C$.

A geometrical discussion of (12) will be presented at some other occasion.

\section{REFERENCES}

1. S. Breuer and D. Gottlieb, The reduction of linear ordinary differential equations with constant coefficients, J. Math. Anal. Appl. 32 (1970), 62-76. MR 41 \#8739.

2. - Explicit characterization of spherical curves, Proc. Amer. Math. Soc. 27 (1971), 126-127. MR 42 \#5165.

3. E. Kreyszig, Differential geometry, Math. Expositions, no. 11, Univ. of Toronto Press, Toronto, 1959. MR 21 \# 7507.

4. E. R. Lorch, A curvature study of convex bodies in Banach spaces, Ann. Mat. Pura Appl. (4) 34 (1955), 105-112. MR 14, 657.

5. R. Nevanlinna, Absolute Analysis, Springer, Berlin, 1959.

6. E. Salkowski, Affine Differentialgeometrie, Teubner, Berlin, 1934.

7. L. A. Santaló, Quelques propriétés des courbes gauches dans la géométrie différentielle affine, Portugal. Math. 3 (1942), 63-68. MR 3, 187.

8. Y.-C. Wong, A global formulation of the condition for a curve to lie in a sphere, Monatsh. Math. 67 (1963), 363-365. MR 27 \#5173.

9. - On an explicit chatacterization of spherical curves, Proc. Amer. Math. Soc. 34 (1972), 239-242; Erratum, ibid. 38 (1973), 668. MR 45 \#4292; 47 \#964. CANADA

DEPARTMENT OF MATHEMATICS, UNIVERSITY OF WINDSOR, WINDSOR, ONTARIO,

DEPARTMENT OF MATHEMATICS, UNIVERSITY OF KARLSRUHE, KARLSRUHE, WEST GERMANY 